\title{
Tunable Dual-Wavelength Thulium-Doped Fiber Laser Based on FBGs and a Hi-Bi FOLM
}

\author{
Manuel Durán-Sánchez, Ricardo I. Álvarez-Tamayo, Berenice Posada-Ramírez, \\ Baldemar Ibarra-Escamilla, Evgeny A. Kuzin, José L. Cruz, and Miguel V. Andrés
}

\begin{abstract}
A tunable dual-wavelength thulium doped fiber laser is demonstrated experimentally. For the first time for the $2-\mu \mathrm{m}$ wavelength band we propose the independent tuning of the generated laser lines based on fiber Bragg gratings and the use of a Hi-Bi fiber optic loop mirror for the fine adjustment of the cavity losses to obtain stable dual-wavelength operation. Dualwavelength laser generation with the laser lines separation in the range from 0.3 to $6.5 \mathrm{~nm}$ is obtained. The laser emission exhibits an optical signal-to-noise ratio better than $56 \mathrm{~dB}$. Improved stability with output power fluctuations less than $1 \mathrm{~dB}$ is observed in dual-wavelength generation with equal power of lines.
\end{abstract}

Index Terms - Fiber lasers, fiber Bragg gratings, Sagnac interferometers, wavelength tuning.

\section{INTRODUCTION}

D UAL-WAVELENGTH fiber lasers based in Tm-doped fibers (TDF) operating at the $2-\mu \mathrm{m}$ wavelength range have been received increasing attention in laser researches due to their great potential applications in optical communication, light detection and ranging (LIDAR), optical signal processing, microwave photonics, terahertz generation, and optical instrumentation. In this sense, dual-wavelength generation of all-fiber Tm-doped fiber lasers (TDFLs) has been increasingly achievable as $2 \mu \mathrm{m}$ compatible fiber components become readily accessible. Recently, different dualwavelength TDFLs approaches at the $2-\mu \mathrm{m}$ wavelength band were reported. These configurations are based on fiber Bragg Gratings (FBG) [1]-[9], spatial mode beating effect [10]-[12], carbon nanotubes [13], photonic crystal fibers [14], and fiber interferometers [1], [15]-[17], among others. Liu et al. reported the single-polarization dual-wavelength generation

The work of M. Durán-Sánchez was supported by CONACyT under Grant 256401. (Corresponding author: Ricardo I. Álvarez-Tamayo.)

M. Durán-Sánchez is with CONACyT-Optics Department, Instituto Nacional de Astrofísica, Óptica y Electrónica, Cholula 72824, México (email:mduransa@conacyt.mx).

R. I. Álvarez-Tamayo is with CONACyT-Universidad Autónoma de Nuevo León, San Nicolás de los Garza 66451, México, (e-mail: rialvarez@ conacyt.mx).

B. Posada-Ramírez, B. Ibarra-Escamilla, and E. A. Kuzin are with the Optics Department, Instituto Nacional de Astrofísica, Óptica y Electrónica, Cholula 72824, México (e-mail: b.posada@inaoep.mx; baldemar@inaoep.mx; ekuz@inaoep.mx).

J. L. Cruz and M. V. Andrés are with the Applied Physics Department, Universidad de Valencia, ICMUV, E-46100 Burjassot, Spain (e-mail: jose.1.cruz@uv.es; miguel.andres@uv.es). of a TDFL based on a polarization maintaining (PM) FBG and a PM Fabry-Perot filter [1]. Zhou et al. proposed a stable dual-wavelength TDFL based on cascaded fiber Bragg gratings [6]. Soltanian et al. achieved stable dual-wavelength operation of a TDFL operating at $1.9 \mu \mathrm{m}$ by using a $10 \mathrm{~cm}$ length of photonic crystal fiber [13]. Nevertheless, most of the previously reported dual-wavelength fiber lasers based on TDF exhibit laser emission with wavelengths below of $2 \mu \mathrm{m}$.

The main issue to achieve the stable dual-wavelength laser generation is the strong mode competition in the homogeneously broadened gain medium. In order to improve the stability of the simultaneously generated laser lines of TDFLs, different techniques for the intracavity losses adjustment have been proposed [1]-[13]. These techniques are based on the use of polarization controllers (PCs) [1]-[7], [10]-[13] and variable optical attenuators [8]-[9]. However, in the proposed techniques a rough and arbitrary adjustment of the intracavity losses is performed which reduces the repeatability and the stability of the dual-wavelength laser generation. From our previous research, the use of a fiber optical loop mirror with a high birefringence fiber in the loop (Hi-Bi FOLM) for the finely adjustment of the intracavity losses has been demonstrated as a reliable, non-arbitrary, and straightforward method to achieve the dual-wavelength generation in erbiumdoped fiber lasers (EDFL) at the $1.55-\mu \mathrm{m}$ waveband [18].

In this letter we propose for the first time, to our knowledge, a TDFL with the tunable dual-wavelength laser generation with wavelength above $2-\mu \mathrm{m}$ with independent wavelengths tuning of both laser lines based on the use of tunable FBGs. We use a Hi-Bi FOLM for intracavity losses adjustment to achieve stable dual-wavelength laser emission.

The dual-wavelength laser emission with maximal wavelengths separations of $6.5 \mathrm{~nm}$ is obtained at the wavelengths of 2067 and $2073.5 \mathrm{~nm}$. The stable dual-wavelength generation with equal powers is obtained with power fluctuations less than $1 \mathrm{~dB}$ and optical signal-to-noise ratio (OSNR) of $\sim 56 \mathrm{~dB}$.

\section{EXPERIMENTAL SETUP}

The schematic of the proposed configuration is shown in Fig. 1(a). A TDF with length of $10 \mathrm{~m}$ is used as a gain medium (TDF, Coractive SCF-TM-8/125). The TDF is pumped by a $1567 \mathrm{~nm}$ fiber laser through a $1550 / 2000 \mathrm{~nm}$ wavelength division multiplexer (WDM). An isolator (ISO) ensures unidirectional propagation from the pump source to the WDM to avoid parasitic lasing and back scattered light to the pumping source. The cavity is formed at one end by 

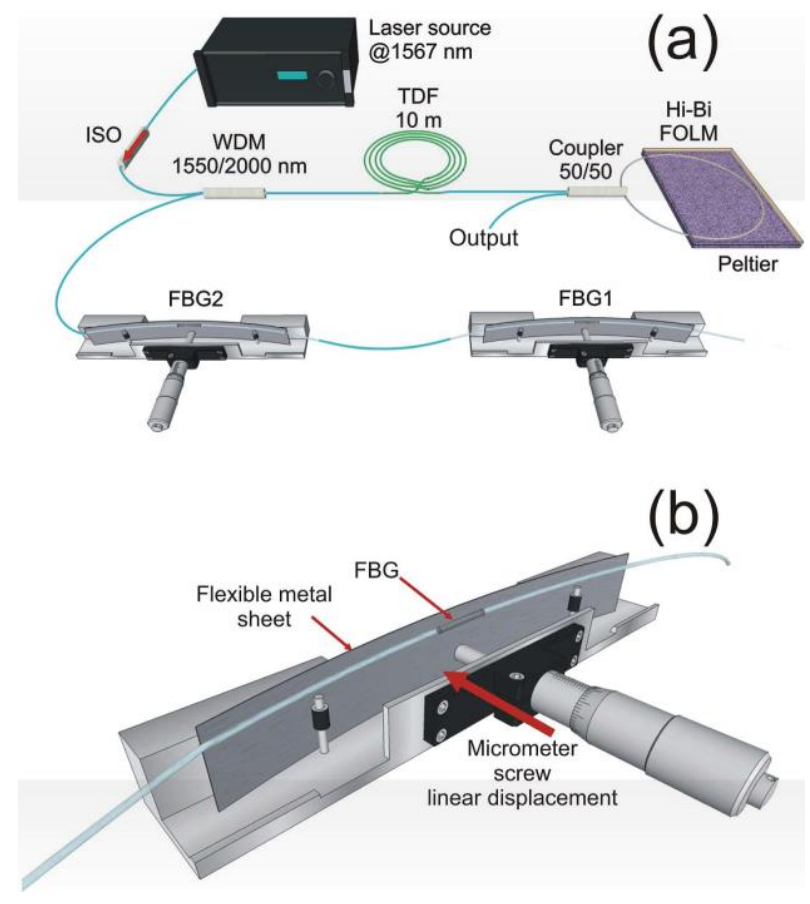

Fig. 1. (a) Experimental setup of the tunable dual-wavelength TDFL, (b) schematic of the tunable FBG filter.

a Hi-Bi FOLM consisting of a 50/50 coupler with output ports connected by a $15-\mathrm{cm}$ of a $\mathrm{Hi}-\mathrm{Bi}$ fiber (birefringence of $4.125 \times 10^{-3}$ ) and a couple of cascaded tunable FBGs at the opposite end. Without strain the FBG1 and FBG2 have 99\% maximum reflection at 2069.16 and $2069.46 \mathrm{~nm}$, respectively. Both FBGs were attached to a thin metal sheet and placed in a mechanical device which applies curvature on the sheet by the linear displacement of the micrometer screw, as it is shown in Fig. 1(b). When the FBG is facing the micrometric screw (position as it is shown in Fig. 1(b)), the internal curvature on the metal sheet causes a compression of the grating resulting in tuning of the Bragg wavelength toward shorter wavelengths. Contrary, when the sheet is flipped to the opposite position, the curvature causes stretching and the Bragg wavelength tunes toward longer wavelengths. The unconnected port of the 50/50 coupler (output) is used to measure the Hi-Bi FOLM transmission spectrum and the laser spectrum by using an optical spectrum analyzer (OSA, Yokogawa AQ6375) with maximal resolution of $0.05 \mathrm{~nm}$.

\section{EXPERIMENTAL RESUlTS AND DISCUSSION}

The Hi-Bi FOLM acts as a broadband reflector with a periodic reflection spectrum. With the used Hi-Bi fiber length the calculated wavelength period of the Hi-Bi FOLM reflection/transmission spectrum in the $2-\mu \mathrm{m}$ region is of $\sim 60 \mathrm{~nm}$. The transmission/reflection spectrum can be displaced by temperature variations of the Hi-Bi fiber [18]. For this, the Hi-Bi fiber loop is placed on a Peltier cooler controlled by an electrical current driver. To measure the transmission period of the Hi-Bi FOLM, the amplified spontaneous emission (ASE) of the TDF was used as input signal when the pump power was below the lasing threshold equal to $125 \mathrm{~mW}$. The signal at

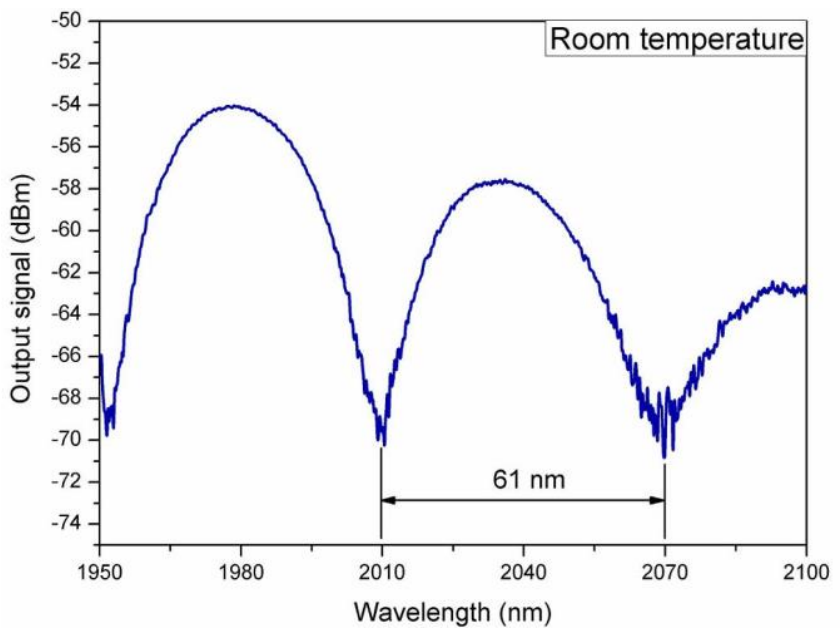

Fig. 2. Output signal of the Hi-Bi FOLM transmission spectrum.

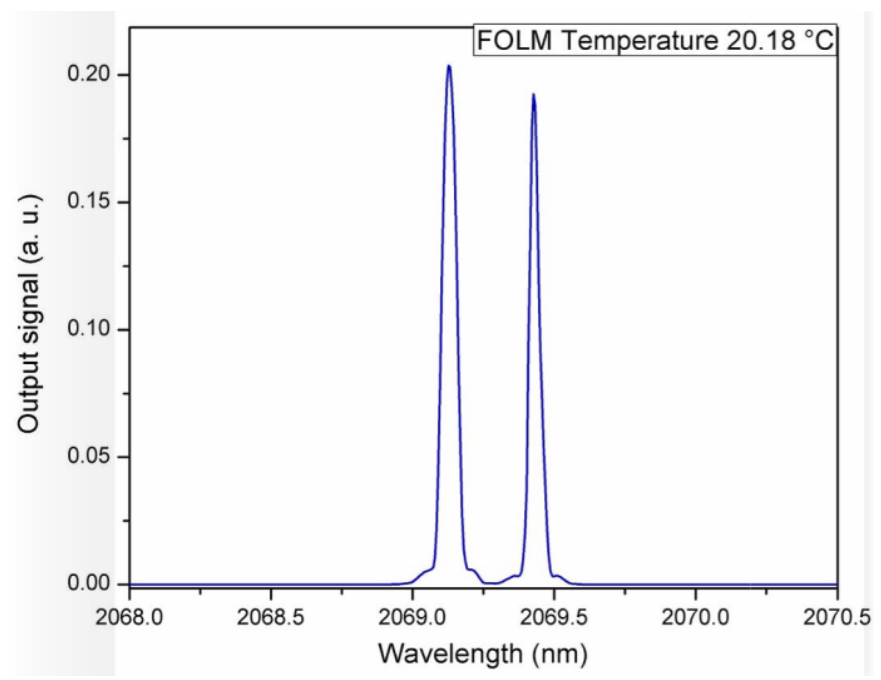

Fig. 3. Output spectrum of the TDFL in dual-wavelength operation.

the Hi-Bi FOLM output is shown in Fig. 2. The measurements were done with the $\mathrm{Hi}-\mathrm{Bi}$ fiber loop at room temperature. The measured period is of $\sim 61 \mathrm{~nm}$. The fringe contrast is more than $15 \mathrm{~dB}$.

With a pump power above $125 \mathrm{~mW}$, the laser emission of the TDFL is reached. In order to achieve dual-wavelength generation, the shift of the Hi-Bi FOLM reflection spectrum to adjust the intracavity losses is performed by temperature variation of the Hi-Bi fiber. Fig. 3 shows the dual-wavelength laser spectrum with equal powers when the maximal pump power of $650 \mathrm{~mW}$ is launched. The Hi-Bi FOLM temperature was adjusted to $20.18{ }^{\circ} \mathrm{C}$. The FBGs were set without strain. The generated laser wavelengths are $2069.16 \mathrm{~nm}$ and $2069.46 \mathrm{~nm}$ with spectral FWHM equal to $60 \mathrm{pm}$ and $37 \mathrm{pm}$ respectively. The wavelengths coincide with the reflection maxima of FBGs.

Fig. 4 shows the measured optical spectrum for dualwavelength operation with equal powers of the TDFL for different laser wavelength separations. The laser lines were tuned by application of stretching to FBG2 and compression to FBG. As it can be observed, the wavelength separation 


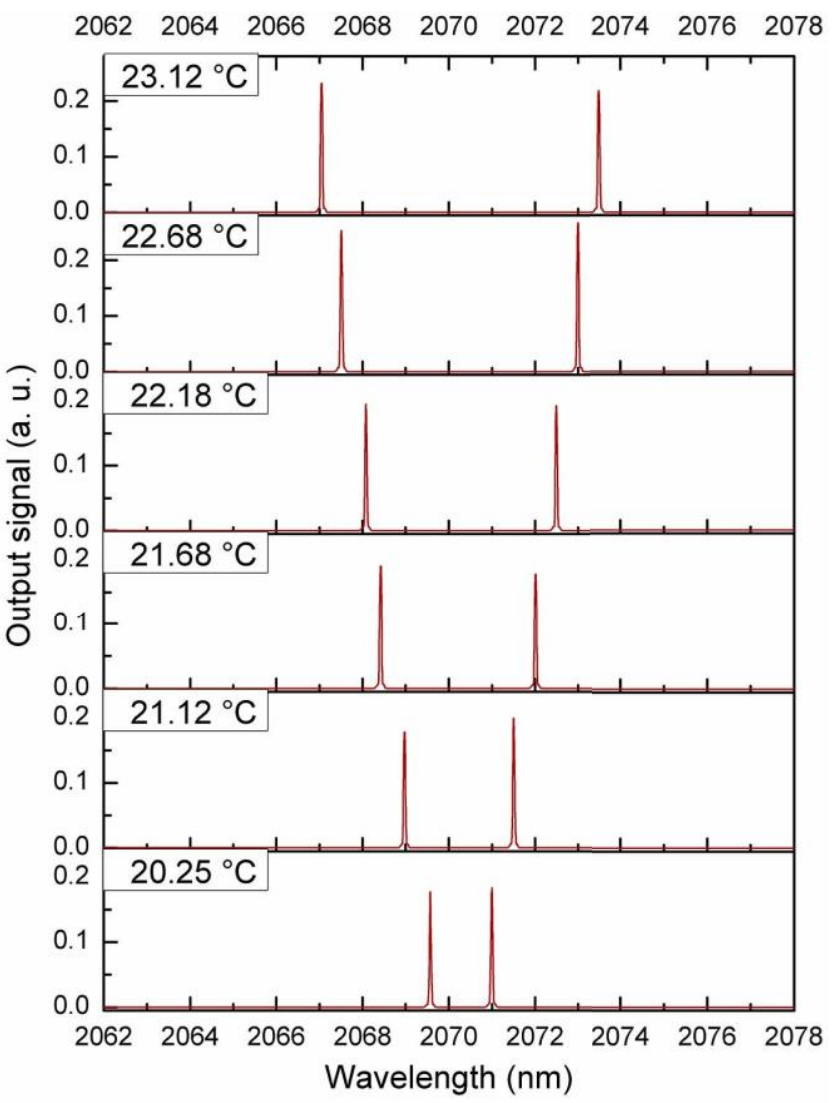

Fig. 4. Tunable dual-wavelength laser operation for different wavelengths separations 2 .

was tuned in the range from 0.3 to $6.5 \mathrm{~nm}$. To obtain dualwavelength operation with approximately equal output powers of the laser lines, the intracavity losses were adjusted by temperature variations of the Hi-Bi FOLM fiber loop. The Hi-Bi loop temperature required for dual wavelength operation with equal output powers was adjusted for each wavelength tuning of FBGs. Initially, the central wavelengths of FBG1 and FBG2 were tuned to 2069.56 and $2071 \mathrm{~nm}$, respectively, to obtain a wavelength separation of $1.5 \mathrm{~nm}$ with a $\mathrm{Hi}-\mathrm{Bi}$ FOLM temperature of $20.25^{\circ} \mathrm{C}$. The wavelength separation was increased in $1 \mathrm{~nm}$ for each measurement until reaching a maximal separation of $6.5 \mathrm{~nm}$ with the Hi-Bi FOLM temperature of $23.12^{\circ} \mathrm{C}$. As it can be observed, with the increase of the wavelength separation between the laser lines, an increase of the Hi-Bi FOLM temperature is required.

The maximal compression applied on FBG1 was of $238 \mu \mathrm{m}$ for a wavelength tuning range from 2069.16 to $2067.05 \mathrm{~nm}$. FBG2 was stretched in the range from 59 to $294 \mu \mathrm{m}$ for a wavelength tuning range from 2070.99 to $2073.52 \mathrm{~nm}$. The wavelength displacement to shorter wavelengths by compression of FBG1 can be linear fitted with a slope of $-96 \mu \mathrm{m} / \mathrm{nm}$. Likewise, the laser wavelength displacement to longer wavelengths by FBG2 stretching slope is of $93.7 \mu \mathrm{m} / \mathrm{nm}$. Fig. 5 shows the mechanical strain applied to the FBGs (by micrometric displacements) to obtain the wavelength separation ( ation results shown in Fig. 4. The Hi-Bi FOLM temperature in which dual-wavelength laser generation with equal powers

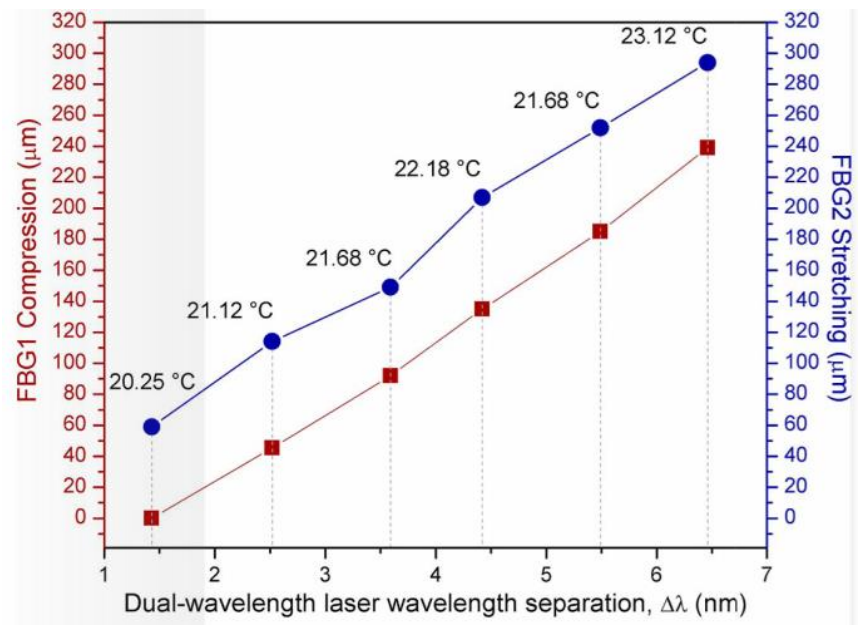

Fig. 5. The FBGs tuning by strain application and the Hi-Bi FOLM temperature adjustment for dual-wavelength laser generation as a function of the dual-wavelength laser lines separation.

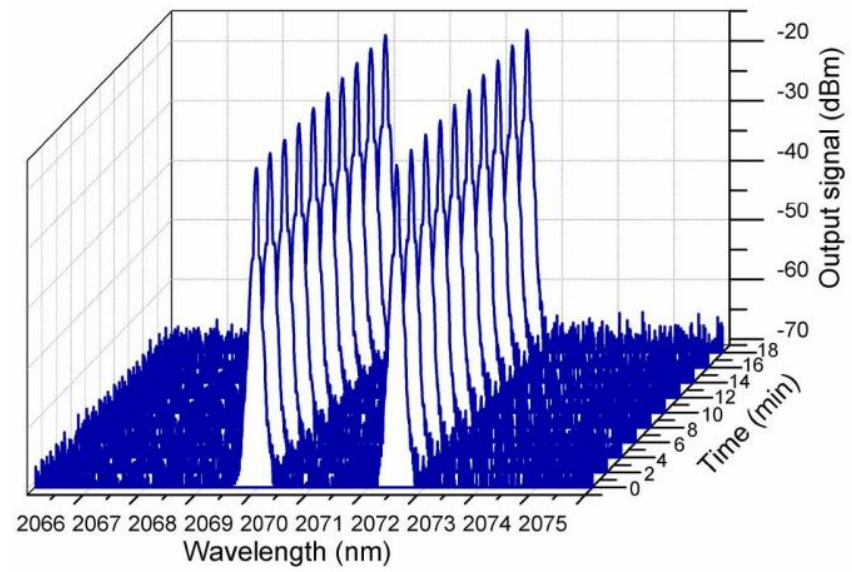

Fig. 6. Stability of the TDFL dual-wavelength generation.

was achieved is also indicated. The Hi-Bi FOLM temperature required to obtain dual-wavelength laser emission with equal powers as a function of can be linear fitted with the slope of $0.56^{\circ} \mathrm{C} / \mathrm{nm}$.

The proposed adjustment of the cavity losses to obtain TDFL dual-wavelength generation with equal powers by temperature variations on the Hi-Bi FOLM fiber loop represents a non-arbitrary method with high finesse of adjustment in which the stability of the simultaneous laser generation is improved due to the fiber loop temperature controlling. The long-time spectrum measurements of the TDFL in dual-wavelength operation with equal powers are shown in Fig 6. The measurements were performed with the maximum pump power of $650 \mathrm{~mW}$. A set of 10 laser spectra were recorded with a 2 minutes interval by the OSA at the output 1 . The temperature of the Hi-Bi FOLM was set at $20.25{ }^{\circ} \mathrm{C}$. The laser lines of the shorter and the longer wavelengths were tuned at 2069 and $2071.5 \mathrm{~nm}$, respectively, to the minimal studied wavelength separation of $1.5 \mathrm{~nm}$. Stable dual-wavelength laser generation is observed. The estimated power fluctuation of both laser wavelengths is less than $0.5 \mathrm{~dB}$. The estimated OSNR for both generated laser wavelengths is greater than $56 \mathrm{~dB}$. 


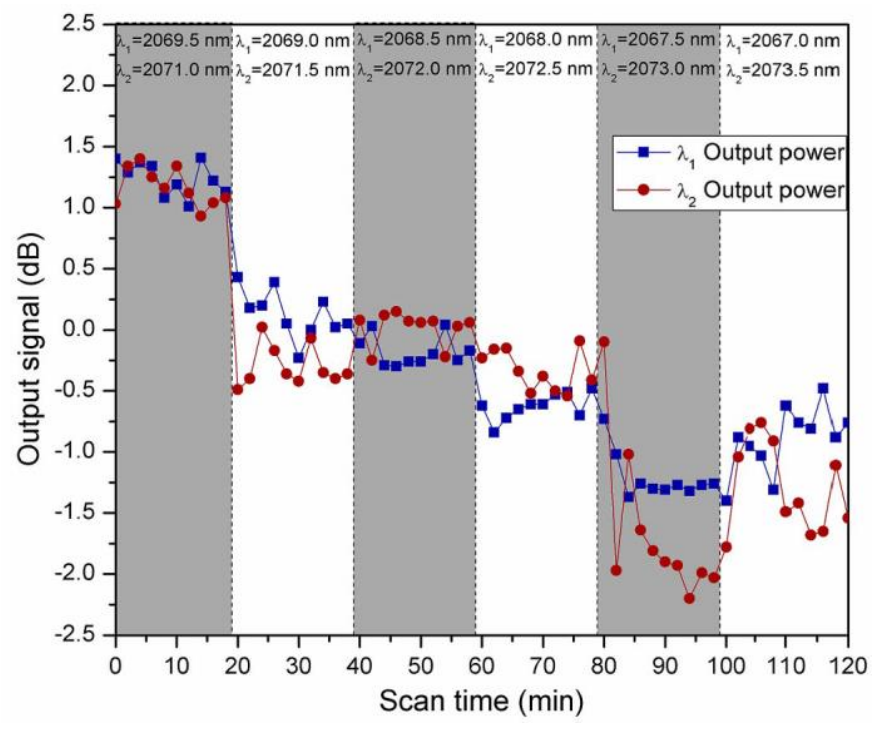

Fig. 7. Output power fluctuations of both laser wavelengths in TDFL dualwavelength operation with equal powers as a function of the wavelength separation between the generated laser lines.

In order to discuss the stability of the TDFL operating in dual-wavelength emission as a function of the wavelength separation between the generated laser lines, a set of 10 laser spectra measurements were obtained with an scan time interval of 2 minutes for each wavelength separation tuned from 1.5 to $6.5 \mathrm{~nm}$ with a step of $1 \mathrm{~nm}$. In Fig. 7 is depicted the output power of both laser lines as a function of the scan time in sets of 10 measurements for each wavelength separation tuning. The wavelength of laser lines for each tuning is also indicated. For the minimum wavelength separation of $1.5 \mathrm{~nm}$, the power fluctuation of both generated laser lines is less than $0.5 \mathrm{~dB}$. For the maximum wavelength separation of $6.5 \mathrm{~nm}$, the maximum power fluctuation of both generated laser lines of $\sim 1 \mathrm{~dB}$ is obtained. However, a relation of the laser lines power fluctuations as a function of the wavelengths separation cannot be established from the obtained results, as it can be observed.

\section{CONCLUSION}

A tunable dual-wavelength TDFL with wavelengths above $2-\mu \mathrm{m}$ with independent wavelengths tuning of both laser lines is experimentally demonstrated. We used tunable FBGs to shift the wavelengths. We proposed for the first time, the use of a Hi-Bi FOLM for fine intracavity losses adjustment to achieve stable dual-wavelength laser emission in a TDFL operating at the $2-\mu \mathrm{m}$ region. Dual-wavelength laser emission with wavelengths separations from 1.5 to $6.5 \mathrm{~nm}$ is obtained. The OSNR of both generated laser lines is $\sim 56 \mathrm{~dB}$. Stable laser emission with power fluctuations less than $1 \mathrm{~dB}$ in dual-wavelength generation with equal powers is obtained.

\section{ACKNOWLEDGMENT}

Manuel Durán-Sánchez and Ricardo I. Álvarez-Tamayo want to thanks to Cátedras CONACyT program.

\section{REFERENCES}

[1] S. Liu et al., "Switchable single-polarization dual-wavelength TDFL using PM Fabry-Perot filter," Opt. Fiber Technol., vol. 29, pp. 1-5, May 2016.

[2] S. Liu, F. Yan, T. Feng, B. Wu, Z. Dong, and G.-K. Chang, "Switchable and spacing-tunable dual-wavelength thulium-doped silica fiber laser based on a nonlinear amplifier loop mirror," Appl. Opt., vol. 53, no. 24, pp. 5522-5526, 2014.

[3] Y. Wang, Y. Zhou, S. Yan, Y. Tang, and J. Xu, "Dual-wavelength 2 $\mu \mathrm{m}$ fiber laser with coupled fiber Bragg grating cavities," IEEE Photon. Technol. Lett., vol. 28, no. 11, pp. 1193-1196, Jun. 1, 2016.

[4] W. J. Peng et al., "1.94 $\mu \mathrm{m}$ switchable dual-wavelength $\mathrm{Tm}^{3+}$ fiber laser employing high-birefringence fiber Bragg grating," Appl. Opt., vol. 52, no. 19, pp. 4601-4607, 2013.

[5] S. Liu, F. Yan, W. Peng, T. Feng, Z. Dong, and G. Chang, "Tunable dualwavelength thulium-doped fiber laser by employing a HB-FBG," IEEE Photon. Technol. Lett., vol. 26, no. 18, pp. 1809-1812, Sep. 15, 2014.

[6] P. Zhou, X. L. Wang, Y. X. Ma, K. Han, and Z. J. Liu, "Stable allfiber dual-wavelength thulium-doped fiber laser and its coherent beam combination," Laser Phys., vol. 21, no. 1, pp. 184-187, 2011.

[7] F. Yan, W. Peng, S. Liu, T. Feng, Z. Dong, and G. K. Chang, "Dualwavelength single-longitudinal-mode Tm-doped fiber laser using PMCMFBG," IEEE Photon. Technol. Lett., vol. 27, no. 9, pp. 951-954, May 1, 2015.

[8] W. Yang et al., "A novel switchable and tunable dual-wavelength singlelongitudinal-mode fiber laser at $2 \mu \mathrm{m}$," IEEE Photon. Technol. Lett., vol. 28, no. 11, pp. 1161-1164, Jun. 1, 2016.

[9] W. Yang, P. Lu, S. Wang, D. Liu, and J. Zhang, " $2-\mu \mathrm{m}$ switchable, tunable and power-controllable dual-wavelength fiber laser based on parallel cavities using $3 \times 3$ coupler," Appl. Phys. B, Lasers Opt., vol. 120, no. 2, pp. 349-354, 2015 .

[10] W. Ma et al., "Wavelength-spacing switchable dual-wavelength single longitudinal mode thulium-doped fiber laser at $1.9 \mu \mathrm{m}$," IEEE Photon. J., vol. 8, no. 6, Dec. 2016, Art. no. 1504508.

[11] M. F. B. Ismail, M. Dernaika, A. Khodaei, S. W. Harun, and H. Ahmad, "Tunable dual-wavelength thulium-doped fiber laser at $1.8 \mu \mathrm{m}$ region using spatial-mode beating," J. Mod. Opt., vol. 62, no. 11, pp. 892-896, 2015.

[12] E. Chen, P. Lu, W. Yang, D. Liu, and J. Zhang, "2- $\mu$ m switchable dualwavelength single-longitudinal-mode fiber laser based on a core-offset structure and carbon nanotube," Appl. Phys. B, Lasers Opt., vol. 122, p. 285 , Nov. 2016.

[13] M. R. K. Soltanian, H. Ahmad, A. Khodaie, I. S. Amiri, M. F. Ismail, and S. W. Harun, "A stable dual-wavelength thulium-doped fiber laser at $1.9 \mu \mathrm{m}$ using photonic crystal fiber," Sci. Rep., vol. 5, Oct. 2015, Art. no. 14537.

[14] W. He, L. Zhu, M. Dong, and F. Luo, "Tuneable and stable multiwavelength thulium-doped ring-cavity fibre laser based on Sagnac loop and Mach-Zehnder filter utilizing thin-core fibre," Laser Phys., vol. 26, no. 12, p. 125102, 2016.

[15] C. Jia, X. Liang, M. Rochette, and L. R. Chen, "Alternate wavelength switching in a widely tunable dual-wavelength $\mathrm{Tm}^{3+}$-doped fiber laser at 1900 nm," IEEE Photon. J., vol. 7, no. 4, Aug. 2015, Art. no. 1502907.

[16] W. He, L. Zhu, M. Dong, and F. Luo, "Tunable and switchable thulium-doped fiber laser utilizing Sagnac loops incorporating two-stage polarization maintaining fibers," Opt. Fiber Technol., vol. 29, pp. 65-69, May 2016.

[17] X. Fan, W. Zhou, S. Wang, X. Liu, Y. Wang, and D. Shen, "Compact dual-wavelength thulium-doped fiber laser employing a double-ring filter," Appl. Opt., vol. 55, no. 12, pp. 3319-3322, 2016.

[18] R. I. Álvarez-Tamayo, M. Durán-Sánchez, O. Pottiez, E. A. Kuzin, B. Ibarra-Escamilla, and A. Flores-Rosas, "Theoretical and experimental analysis of tunable Sagnac high-birefringence loop filter for dualwavelength laser application," Appl. Opt., vol. 50, no. 3, pp. 253-260, 2011. 